\title{
The mitochondrial origin of postischemic arrhythmias
}

\author{
Fadi G. Akar, Miguel A. Aon, Gordon F. Tomaselli, and Brian O'Rourke \\ Division of Cardiology, Johns Hopkins University School of Medicine, Baltimore, Maryland, USA.
}

\begin{abstract}
Recovery of the mitochondrial inner membrane potential $\left(\Delta \Psi_{\mathrm{m}}\right)$ is a key determinant of postischemic functional recovery of the heart. Mitochondrial ROS-induced ROS release causes the collapse of $\Delta \Psi_{\mathrm{m}}$ and the destabilization of the action potential (AP) through a mechanism involving a mitochondrial inner membrane anion channel (IMAC) modulated by the mitochondrial benzodiazepine receptor ( $\mathrm{mBzR})$. Here, we test the hypothesis that this mechanism contributes to spatiotemporal heterogeneity of $\Delta \Psi_{\mathrm{m}}$ during ischemia-reperfusion (IR), thereby promoting abnormal electrical activation and arrhythmias in the whole heart. High-resolution optical AP mapping was performed in perfused guinea pig hearts subjected to 30 minutes of global ischemia followed by reperfusion. Typical electrophysiological responses, including progressive AP shortening followed by membrane inexcitablity in ischemia and ventricular fibrillation upon reperfusion, were observed in control hearts. These responses were reduced or eliminated by treatment with the mBzR antagonist 4'-chlorodiazepam (4'-Cl-DZP), which blocks depolarization of $\Delta \Psi_{\mathrm{m}}$. When applied throughout the IR protocol, 4'-Cl-DZP blunted AP shortening and prevented reperfusion arrhythmias. Inhibition of ventricular fibrillation was also achieved by bolus infusion of 4'-Cl-DZP just before reperfusion. Conversely, treatment with an agonist of the $\mathrm{mBzR}$ that promotes $\Delta \Psi_{\mathrm{m}}$ depolarization exacerbated IR-induced electrophysiological changes and failed to prevent arrhythmias. The effects of these compounds were consistent with their actions on IMAC and $\Delta \Psi_{\mathrm{m}}$. These findings directly link instability of $\Delta \Psi_{\mathrm{m}}$ to the heterogeneous electrophysiological substrate of the postischemic heart and highlight the mitochondrial membrane as a new therapeutic target for arrhythmia prevention in ischemic heart disease.
\end{abstract}

\section{Introduction}

Ischemia-reperfusion (IR) of the heart leads to many biochemical, ion homeostasis, and ion channel alterations that may contribute to postischemic contractile and electrical dysfunction and serve as a substrate for fatal arrhythmias (1). Several mechanistic hypotheses, such as extracellular $\mathrm{K}^{+}$accumulation (2), depression of gapjunctional conductance (3), and dispersion of action potential (AP) repolarization (4), have emerged as dominant paradigms to explain the genesis of arrhythmias upon reperfusion, but the sequence of cellular events underlying postischemic electrical instability has not been elucidated. Sarcolemmal ATP-sensitive $\mathrm{K}^{+}\left(\mathrm{K}_{\mathrm{ATP}}\right)$ channels are thought to mediate AP shortening during ischemia and may contribute to postischemic electrical heterogeneity, but how these channels are activated and whether they contribute to or retard functional electrical recovery upon reperfusion are unresolved issues.

Because $\mathrm{K}_{\mathrm{ATP}}$ channels are metabolic sensors, their role in postischemic electrical dysfunction is likely to depend on mitochondrial bioenergetics. Various forms of metabolic stress lead to depolarization of the mitochondrial inner membrane potential (5-8), and postischemic conditions, including cellular $\mathrm{Ca}^{2+}$ overload and

Nonstandard abbreviations used: AP, action potential; APA, action potential amplitude; APD, action potential duration; BzR, benzodiazepine receptor; 4'-Cl-DZP, 4'-chlorodiazepam; CsA, cyclosporin A; CV, conduction velocity; $d F / d t$, AP upstroke velocity; FGIN-1-27, N,N-Dihexyl-2-(4-fluorophenyl)indole-3-acetamide; GLIBEN, glibenclamide; IMAC, inner membrane anion channel; IR, ischemia-reperfusion; $\mathrm{K}_{\mathrm{ATP}}$, ATP-sensitive $\mathrm{K}^{+}$(channels); $\mathrm{mBzR}$, mitochondrial BzR; PTP, permeability transition pore; $\Delta \Psi_{\mathrm{m}}$, recovery of mitochondrial inner membrane potential; TMRM, tetramethylrhodamine methyl ester; VF, ventricular fibrillation; VT, ventricular tachycardia. Conflict of interest: The authors have declared that no conflict of interest exists.

Citation for this article: J. Clin. Invest. 115:3527-3535 (2005).

doi:10.1172/JCI25371. an increase in the production of ROS, favor the degradation of mitochondrial integrity (9-11), leading to necrotic or apoptotic cell death (12). The activation of energy-dissipating channels on the inner membrane, including the mitochondrial permeability transition pore (PTP), has been proposed to mediate cell death during reperfusion (13-15); however, other studies have shown that the PTP inhibitor cyclosporin A (CsA) delays but does not prevent the loss of mitochondrial inner membrane potential $\left(\Delta \Psi_{\mathrm{m}}\right)$ in the postischemic heart (16).

We have previously demonstrated that metabolic stress in the form of substrate deprivation $(7,17)$ or localized ROS generation (8) can trigger cell-wide oscillations or collapse of $\Delta \Psi_{\mathrm{m}}$ in isolated cardiomyocytes. This provided evidence in support of a direct connection between loss of mitochondrial function, the $\mathrm{K}_{\mathrm{ATP}}$ channel, and alterations in the cellular AP. The rapid uncoupling of oxidative phosphorylation during depolarization of $\Delta \Psi_{\mathrm{m}}$ was closely linked to the activation of sarcolemmal $\mathrm{K}_{\mathrm{ATP}}$ currents, consequently shortening the cellular AP and rendering the myocyte electrically inexcitable during the nadir of $\Delta \Psi_{\mathrm{m}}$ oscillation (8). We proposed that this mechanism could contribute to destabilization of AP repolarization during IR in the whole heart, possibly leading to arrhythmias (18). Further investigation into the mechanism of this phenomenon revealed that several inhibitors of the mitochondrial inner membrane anion channel (IMAC), including antagonists of the mitochondrial benzodiazepine receptor ( $\mathrm{mBzR}$ ) (18), could reversibly suppress or prevent the mitochondrial ROSinduced ROS release response, thereby stabilizing $\Delta \Psi_{\mathrm{m}}$ (8). Importantly, the PTP was not activated during the oscillations in $\Delta \Psi_{\mathrm{m}}$ (8), and CsA was ineffective in preventing the loss of $\Delta \Psi_{\mathrm{m}}(7,8)$. A mechanistic scheme involving the activation of IMAC by ROS was also supported by a computational model (19). 
A

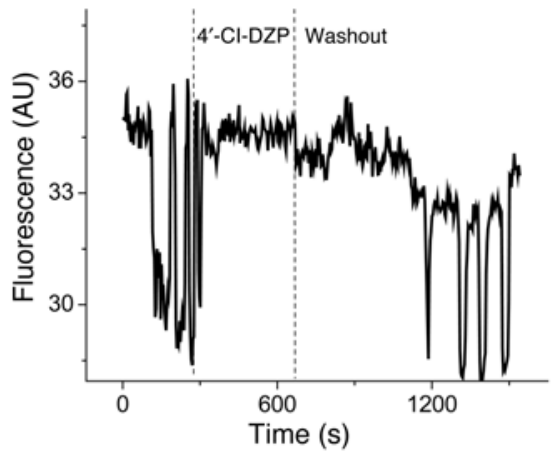

B

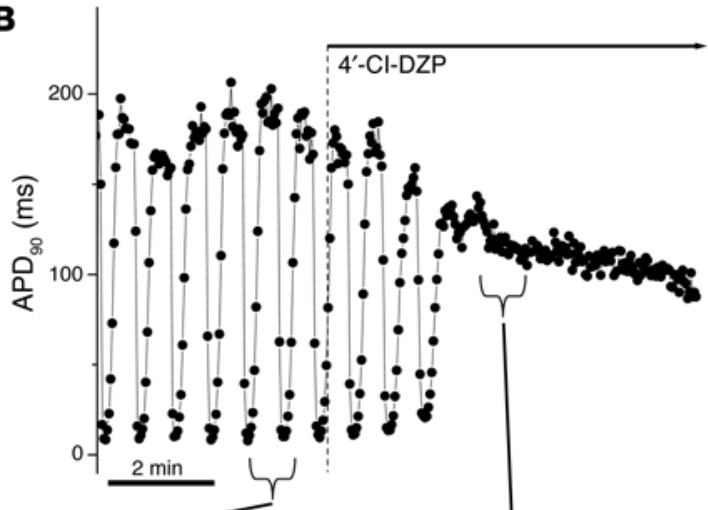

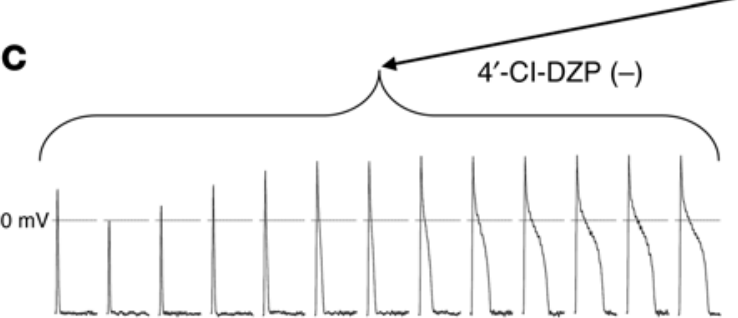
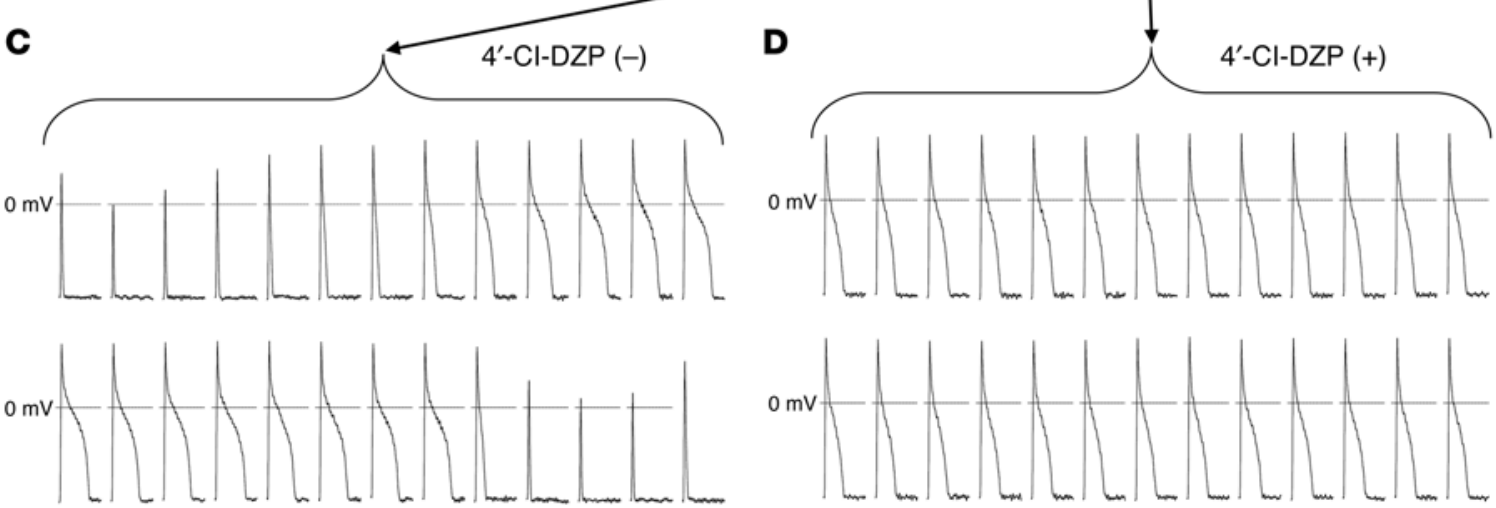

Figure 1

Blockade of mitochondrial oscillations and stabilization of the cellular AP by 4'-Cl-DZP. Freshly isolated cardiomyocytes were loaded with TMRM $(100 \mathrm{nM})$ at $37^{\circ} \mathrm{C}$, and APs were recorded under whole-cell current-clamp conditions on the stage of the microscope as described in Methods. (A) The reversible effect of acutely added 4'-Cl-DZP (32 $\mu \mathrm{M})$ on mitochondrial $\Delta \Psi_{\mathrm{m}}$ oscillations. (B) Mitochondrial oscillations in $\Delta \Psi_{\mathrm{m}}$ and the sarcolemmal APD were triggered after a highly localized laser flash (3 minutes before the train of oscillating APDs shown in this panel). APs evoked by brief current injections were recorded during the oscillations. Previously, we showed that during a synchronized cell-wide depolarization-repolarization cycle, the AP shortens in synchrony with fast mitochondrial depolarization (4). (C) During the APD oscillations, the cell becomes inexcitable when $\Delta \Psi_{\mathrm{m}}$ is fully depolarized (remaining upward spikes are from the stimulus only). (D) After addition of $64 \mu \mathrm{M} 4^{\prime}$-Cl-DZP, a stable AP is restored and $\Delta \Psi_{m}$ oscillations are suppressed.

Here, we test the hypothesis that IR-related arrhythmias can be suppressed by prevention of the collapse of mitochondrial function through inhibition of the $\mathrm{mBzR}$. Utilizing high-resolution optical AP mapping, we show that $\mathrm{mBzR}$ inhibition reduces AP shortening during ischemia, prevents ventricular fibrillation (VF), and facilitates the restoration of AP duration (APD) upon reperfusion. These effects were consistent with the stabilization of $\Delta \Psi_{\mathrm{m}}$ and the cellular AP when the $\mathrm{mBzR}$ antagonist was applied to isolated cardiac cells. These findings suggest a novel mechanism for ischemia-related arrhythmias and identify an important new therapeutic target for the prevention of postischemic electrical dysfunction.

\section{Results}

$m B z R$ antagonist stabilizes $\Delta \Psi_{m}$ and the cellular $A P$. To demonstrate a mechanistic link between the mitochondrial energy state and electrical excitability, we used a previously described method for triggering whole-cell oscillations in $\Delta \Psi_{\mathrm{m}}$ by focal 2-photon laser excitation, in which laser-induced depolarization of a few mitochondria leads to a sustained autonomous oscillation in the entire mitochondrial network (8). $\Delta \Psi_{\mathrm{m}}$, reported by tetramethylrhodamine methyl ester (TMRM) fluorescence, was imaged while APs were simultaneously recorded using the patch-clamp technique in current-clamp mode (Figure 1). The mBzR antagonist 4'-chlorodiazepam (4'-Cl-DZP) suppressed whole-cell oscillations in $\Delta \Psi_{\mathrm{m}}$ within minutes of application, and the effect was reversible, as $\Delta \Psi_{\mathrm{m}}$ oscillations returned within 10 minutes of washout of the compound (Figure 1A). Through its stabilizing effect on $\Delta \Psi_{\mathrm{m}}$, 4 '-Cl-DZP also eliminated oscillations in APD (Figure 1B). In the absence of the drug, $\mathrm{APD}_{90}$ decreased to an electrically inexcitable state within approximately 4 seconds $(2-3$ stimuli at $0.5 \mathrm{~Hz})$ during each cycle of mitochondrial depolarization. Within approximately 2 minutes of drug application ( $64 \mu \mathrm{M}$; Figure 1, B and D), AP oscillations were suppressed and $\mathrm{APD}_{90}$ stabilized.

We have used the term mitochondrial criticality to refer to the state of the mitochondrial network just prior to cell-wide depolarization of $\Delta \Psi_{\mathrm{m}}$, when the system becomes very sensitive to even small perturbations in conditions (20). Since an elevation of ROS and loss of $\Delta \Psi_{\mathrm{m}}$ also occurs during IR of the intact heart, we next tested whether mitochondrial criticality might contribute to alterations in the electrophysiological substrate leading to postischemic arrhythmias in the intact heart.

Baseline electrophysiological properties. In order to investigate whether mitochondrial ROS-induced activation of IMAC underlies the electrical dysfunction of hearts subjected to IR, we established a proto- 


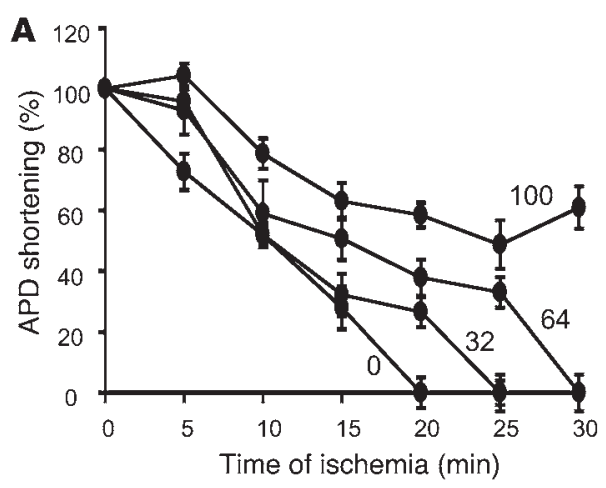

\section{Figure 2}

Protection against ischemia-induced APD shortening by 4'-Cl-DZP. (A) Ischemia-induced APD shortening in control hearts and hearts treated with varying concentrations of 4'-Cl-DZP. (B) Representative APs from a control and a 100 $\mu \mathrm{M}$ 4'-Cl-DZP-treated heart recorded at various intervals during the ischemia protocol. At baseline, APD of control and $100 \mu \mathrm{M}$ 4'-Cl-DZP-treated hearts were comparable. col for reproducibly inducing VF in Langendorff-perfused guinea pig hearts. To rule out direct effects of the compounds used in the study on sarcolemmal ion channels, we first determined whether 4'-Cl-DZP, CsA, N,N-Dihexyl-2-(4-fluorophenyl)indole-3-acetamide (FGIN-1-27), or glibenclamide (GLIBEN) altered intrinsic electrophysiological properties, including APD and conduction velocity $(\mathrm{CV})$, during baseline perfusion. None of the compounds had a significant impact on baseline APD $(186 \pm 18,174 \pm 16,168 \pm 24$, $188 \pm 16$ ms, $182 \pm 16$; control, FGIN-1-27, CsA, 4'Cl-DZP, GLIBEN, respectively) or CV $(41 \pm 4,44 \pm 6,40 \pm 4,39 \pm 5,41 \pm 5 \mathrm{~cm} / \mathrm{s})$ at the optimal concentration for each agent used in this study $(4.6 \mu \mathrm{M}$ FGIN-1-27, $0.2 \mu \mathrm{M}$ CsA, $64 \mu \mathrm{M}$ 4'Cl-DZP, and $10 \mu \mathrm{M}$ GLIBEN). At a relatively high concentration $(46 \mu \mathrm{M})$, the $\mathrm{mBzR}$ agonist FGIN-1-27, which we have previously shown to induce $\Delta \Psi_{\mathrm{m}}$ depolarization (8), produced significant APD shortening and membrane inexcitability that occurred within 15 minutes of drug delivery during normal perfusion (results not shown). The highest concentration $(100 \mu \mathrm{M})$ of $4^{\prime} \mathrm{Cl}$-DZP tested had no significant effect on $\mathrm{APD}$ or $\mathrm{CV}$ during baseline perfusion and was the most effective in blunting AP shortening during ischemia (see below); however, it was not as effective as the lower concentration of $64 \mu \mathrm{M}$ in restoring the APD upon reperfusion and preventing arrhythmias. Hence the lower concentration was selected as the optimal concentration to be used in subsequent experiments.

Response to ischemia. We then investigated whether any of these pharmacological interventions modulated the electrophysiological response of hearts to no-flow global ischemia. As expected, ischemia resulted in progressive shortening of APD (Figure 2), resulting in complete loss of AP after $18.4 \pm 3.3$ minutes under control conditions (i.e., without pharmacological intervention prior to the ischemic episode). Also as expected, APD shortening in ischemia was accompanied by progressive reduction of the AP upstroke velocity $(d F / d t)$, which occurred over a similar time frame (measured as a change in the rate of dye fluorescence).

Interestingly, treatment with the $\mathrm{mBzR}$ antagonist had a profound influence on the electrophysiological response to ischemia. 4'-ClDZP blunted APD shortening in a dose-dependent fashion during ischemia, consistent with its proposed role in preventing mitochondrial depolarization and the subsequent activation of sarcolemmal $\mathrm{K}_{\mathrm{ATP}}$ currents (Figure 2A). Remarkably, at the highest concentration of 4'-Cl-DZP tested $(100 \mu \mathrm{M})$, APs persisted even after 30 minutes of no-flow ischemia, a time when all of the untreated ischemic hearts became electrically inexcitable (Figure 2B).

Conversely, pretreatment of hearts with the mBzR agonist FGIN1-27 accelerated APD shortening during ischemia and significantly reduced the time for the onset of inexcitability $(12.7 \pm 2.6$ minutes $)$ compared with control hearts ( 18 minutes, Figure $3 \mathrm{~A})$. In addition, FGIN-1-27 treatment was associated with disproportionate slowing of CV (see Supplemental Video 1, at 5 minutes; supplemental material available online with this article; doi:10.1172/ JCI25371DS1) compared with untreated hearts (Supplemental Video 2, at 5 minutes). Such profound CV slowing in FGIN-1-27treated hearts was shortly followed by the emergence of an area of functional conduction block within as little as 10 minutes of
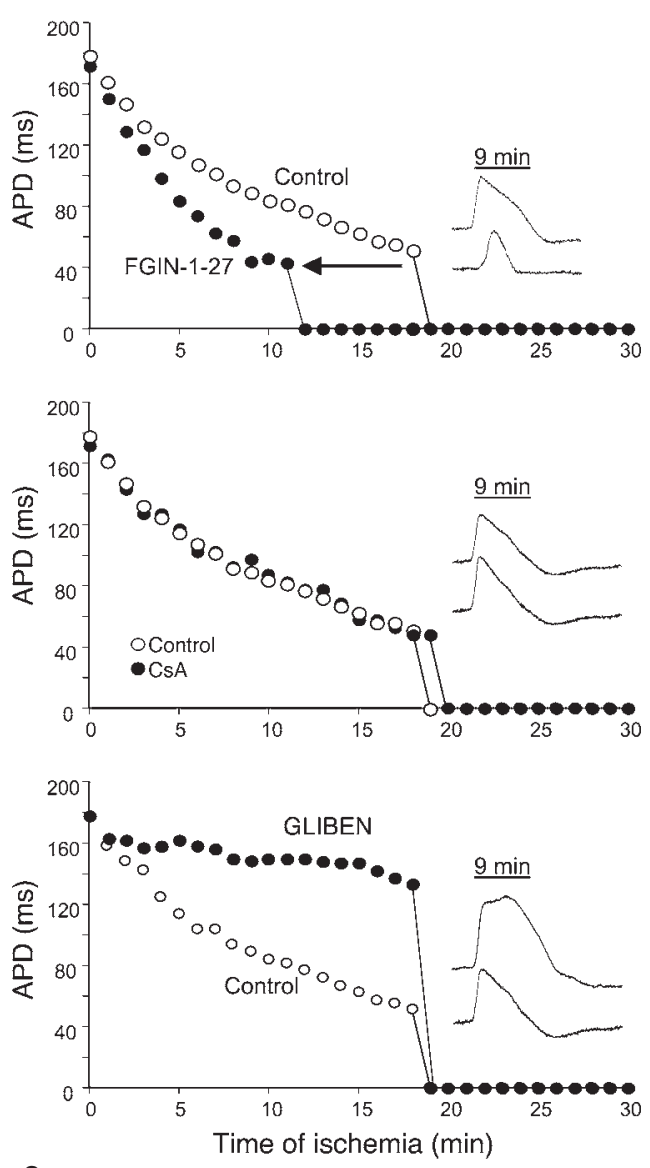

Figure 3

Ischemia-induced APD shortening and representative APs recorded from hearts pretreated with 4.6 $\mu \mathrm{M}$ FGIN-1-27 (top panel), $0.2 \mu \mathrm{M}$ CsA (middle panel), and $10 \mu \mathrm{M}$ GLIBEN (bottom panel) compared with control untreated hearts. 

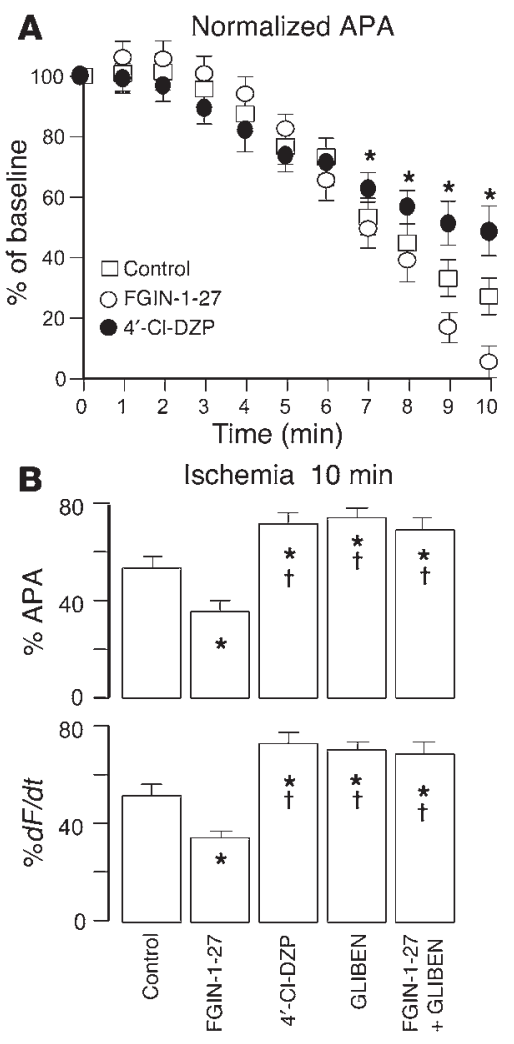

C
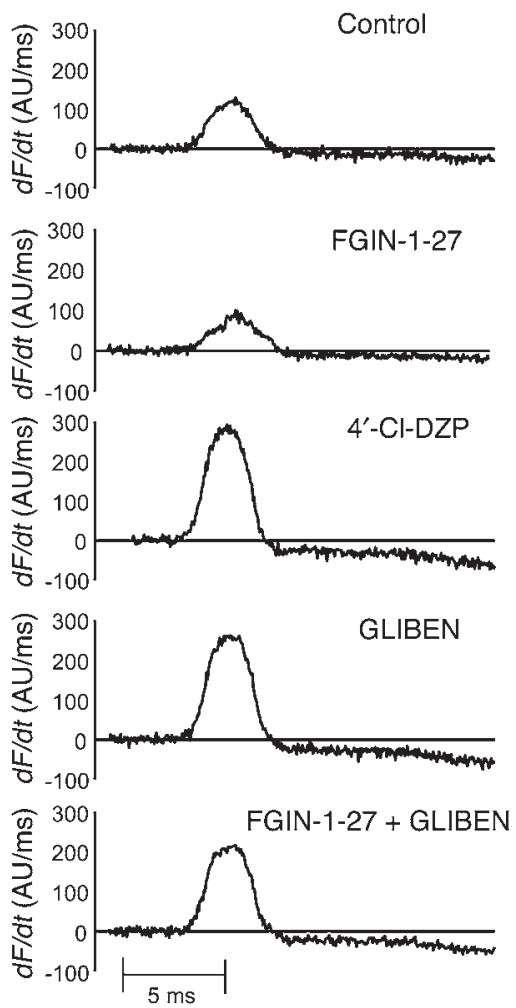

\section{Figure 4}

Effects on ischemia-induced AP amplitude and upstroke velocity. (A) Progressive reduction in the APA during the first 10 minutes of ischemia in control, FGIN-1-27-, and 4'-Cl-DZP-treated hearts. FGIN-1-27-treated hearts exhibited a more enhanced reduction of APA compared with control and $4^{\prime} \mathrm{Cl}$-DZP-treated hearts. (B) Comparison of normalized APA and $d F / d t$ after 10 minutes of ischemia compared with preischemic baseline perfusion in untreated control hearts and hearts treated with FGIN-1-27, 4'-CI-DZP, GLIBEN, or a combination of FGIN-1-27 and GLIBEN. ${ }^{*} P<0.05$ vs. control; $\dagger P<0.05$ vs. FGIN-1-27. (C) Representative raw traces of $d F / d t$ in control hearts and hearts treated with FGIN-1-27, 4'-CI-DZP, GLIBEN, or a combination of FGIN-1-27 and GLIBEN. ischemia (Supplemental Video 1, at 10 minutes) while in control hearts, conduction was slowed but persisted beyond 10 minutes of ischemia (Supplemental Video 2, at 10 minutes).

Importantly, inhibition of the mitochondrial PTP with CsA did not have an impact on the response of hearts to ischemia, as APD shortening and onset of inexcitability were not significantly altered compared with control hearts (Figure 3B). In accord with the hypothesis that mitochondrial uncoupling was linked to sarcolemmal $\mathrm{K}_{\mathrm{ATP}}$ channel activation, the $\mathrm{K}_{\mathrm{ATP}}$ channel inhibitor GLIBEN caused a marked reduction in the rate of APD shortening with ischemia up to a point when the heart abruptly became inexcitable at approximately 18 minutes (Figure 3C). Interestingly, the time to inexcitability was similar to that of control hearts despite robust preservation of APD by GLIBEN at earlier time points (Figure 3C).

Ischemic elevation of extracellular $\mathrm{K}^{+}$concentration, which may be mediated in part by $\mathrm{K}_{\mathrm{ATP}}$ channel opening, might also be expected to partially depolarize the cellular resting membrane potential. This effect can be indirectly assessed by examining the AP amplitude (APA) and $d F / d t$, since these parameters are predominantly determined by the extent of inactivation of $\mathrm{Na}^{+}$currents due to depolarization of the resting membrane potential. Therefore, in addition to measuring the marked changes in APD and the time to onset of inexcitability, we quantified ischemia-induced changes in the normalized APA (relative to the preischemia baseline level in each heart) and $d F / d t$ in control hearts and hearts treated with FGIN-1-27, 4'Cl-DZP, GLIBEN, and a combination of FGIN-1-27 and GLIBEN. As expected, ischemia caused progressive reduction of normalized APA (Figure $4 \mathrm{~A}$ ) and $d F / d t$. Ischemia-induced reduction of APA and $d F / d t$ was highly sensitive to treatment with FGIN1-27, 4'Cl-DZP, and GLIBEN (Figure 4, B and C). While FGIN-1-27 accentuated the ischemia-induced reduction of APA and $d F / d t$, treatment with $4^{\prime}$-Cl-DZP and GLIBEN protected against such decrease in both parameters relative to control hearts (Figure 4, B and C). Finally, treatment with GLIBEN was also effective in abolishing the FGIN-1-27-induced reduction of APA and $d F / d t$ when the heart was treated with both compounds.

There was a more pronounced reduction in membrane excitability during ischemia in hearts treated with FGIN-1-27. Figure 5 shows a sequence of isopotential contour maps recorded every $1.2 \mathrm{~ms}$ that demonstrates the sequential spread of the AP wavefront (depolarized myocardium shown in red and resting myocardium shown in blue) across the epicardial surface of a representative heart treated with FGIN-1-27 after 11 minutes of ischemia. Also shown (Figure $5 \mathrm{~B}$ ) are representative $\mathrm{AP}$ traces recorded at 11 minutes of ischemia (left) and following 10 minutes of reperfusion (right). Clearly, 11 minutes of ischemia resulted in conduction block (upper left corner of isopotential contour maps), as the depolarization wavefront failed to propagate into this electrically silent area. Importantly, these areas of electrical silence were also present at the same location in the heart during reperfusion and likely participated in the formation of sustained arrhythmias (Figure 5B, right). A similar pattern of conduction block upon reperfusion is shown dynamically in another heart treated with FGIN-1-27 (Supplemental Video 3).

Response to reperfusion. After characterizing the ischemia-induced electrophysiological changes, we next investigated the response of hearts to reperfusion following the 30-minute ischemic episode with and without pretreatment with 4'-Cl-DZP, FGIN-1-27, CsA, GLIBEN, or a combination of FGIN-1-27 and GLIBEN. Reperfusion of untreated control hearts was associated with sustained VF in $89 \%$ of hearts (Figure 6 ).

In addition to preventing ischemia-induced APD shortening, treatment of hearts with 4'-Cl-DZP promoted the rapid recovery 


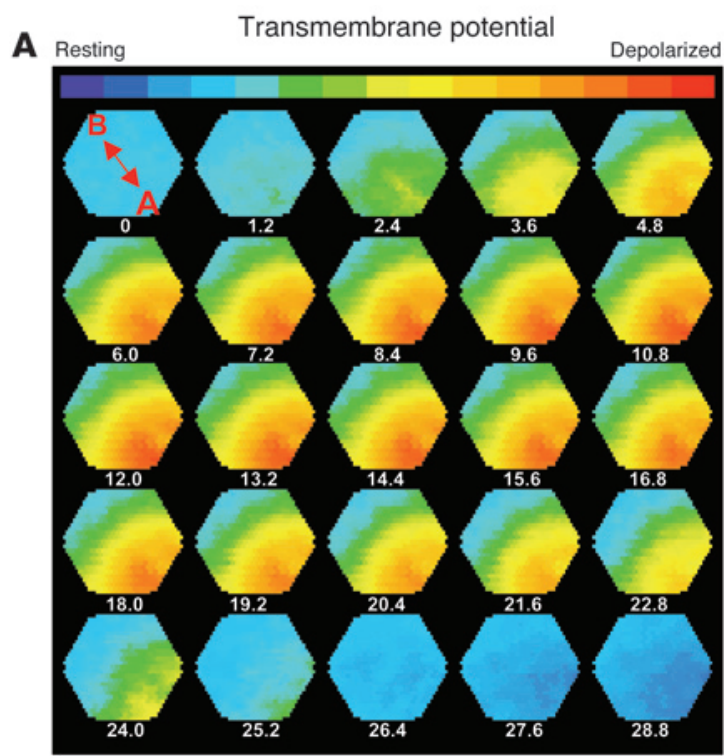

B

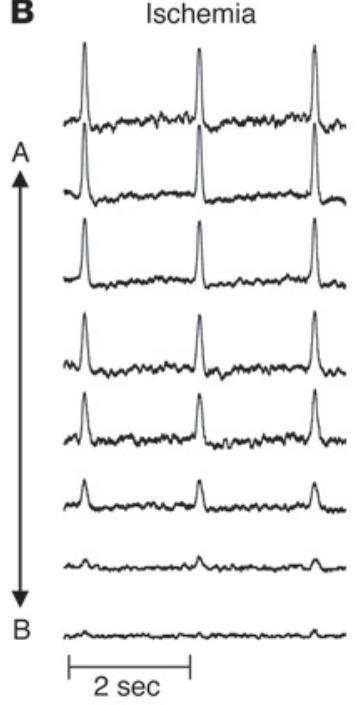

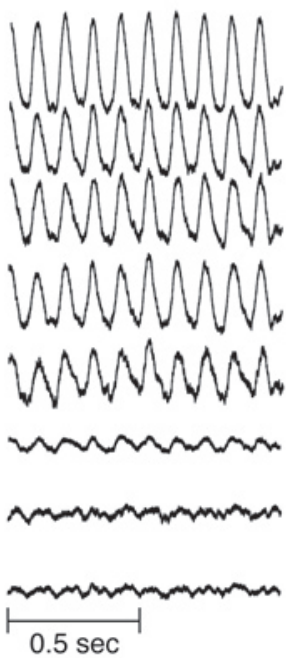

Reperfusion

of AP morphology and APD upon reperfusion (Figures 6B and 7A) and markedly decreased the incidence of postischemic arrhythmias (10 of 12 hearts exhibited no sustained arrhythmias; Figure 6A). Prevention of reperfusion-related VF was evident both when 4'-ClDZP (32-64 $\mu \mathrm{M})$ was delivered continuously prior to ischemia (6 of 8 ) and when it was given in a high dose $(320 \mu \mathrm{M})$ bolus ( 4 of 4$) 5$ minutes prior to the onset of reperfusion (Figure 6A).

In contrast, FGIN-1-27 treatment resulted in a prolonged period of electrical silence upon reperfusion, followed by ventricular tachycardia (VT) in all (6 of 6) treated hearts (Figures 6A and 7A). Compared with control hearts, VT presented with a significantly longer cycle length in FGIN-1-27-treated hearts compared with polymorphic VT/VF in normal hearts (not shown).

Treatment with $0.2 \mu \mathrm{M}$ CsA was also associated with a markedly $(P<0.01)$ longer period of electrical inexcitability upon reperfusion (8 minutes for CsA compared with less than 2 minutes for 4'-Cl-DZP), followed by a slower, partial recovery of the AP as compared with that in 4'-Cl-DZP-pretreated hearts (Figure 7, A and C). This concentration of CsA was chosen based on an earlier report

\section{Figure 5}

Metabolic sink/block as a mechanism of conduction failure and arrhythmias. (A) Sequential isopotential contour maps recorded every $1.2 \mathrm{~ms}$ that display the level of membrane potential (color coded) across 464 epicardial sites simultaneously. Red indicates depolarized membrane potential, and blue indicates resting membrane potential. These maps demonstrate the sequential spread of activation across the epicardium of a representative guinea pig heart treated with FGIN-1-27 at 11 minutes of ischemia. Under these conditions, the wavefront fails to propagate across the entire mapping field (i.e., metabolic sink/block). (B) Representative AP traces (A to B, location marked on first contour map) recorded at 11 minutes of ischemia (left) and 10 minutes of reperfusion (right) indicating presence of conduction block (left) and arrhythmias with electrical silence at the same sites of conduction block (right).

that demonstrated that it was optimal for inhibiting PTP in the reperfused heart (14). This was confirmed by further experiments using higher $(0.4,1 \mu \mathrm{M})$ or lower $(0.1 \mu \mathrm{M})$ CsA concentrations, which resulted in diminished AP recovery (Figure $7 \mathrm{~B}$ ) and a higher incidence of VT/VF (Figure 6A).

\section{Discussion}

The present findings support the hypothesis that IR-related electrophysiological alterations and arrhythmias in intact hearts are in part a consequence of the failure of the cellular mitochondrial network to maintain $\Delta \Psi_{\mathrm{m}}$. The major findings of the study are that preventing $\Delta \Psi_{\mathrm{m}}$ depolarization by blocking the $\mathrm{mBzR}$ (a) stabilizes the AP of metabolically-stressed cardiomyocytes, (b) blunts ischemia-induced AP shortening, (c) improves postischemic recovery of the AP, and (d) prevents the occurrence of spontaneous arrhythmias upon reperfusion in the whole heart. Conversely, facilitating $\Delta \Psi_{\mathrm{m}}$ depolarization with an $\mathrm{mBzR}$ agonist accelerates ischemiainduced AP changes, creates large regions of conduction block, and promotes sustained stable arrhythmias upon reperfusion.

Role of $m B z R$ in ROS-dependent mitochondrial $\Delta \Psi_{m}$ oscillations in heart cells. We have previously described in experimental (8) and theoretical (19) studies a mechanism whereby the mitochondrial network of the cardiac cell is pushed to a critical state (20) by metabolic or oxidative stress. The mechanism involves the regenerative activation of IMAC by mitochondrial ROS-induced ROS release. Mitochondrial depolarization could be suppressed either by inhibiting the production of ROS by the electron transport chain, enhancing the ROS scavenging capacity of the cell, or blocking IMAC by means of anion transport inhibitors or $\mathrm{mBzR}$ antagonists $(19,20)$. Oscillations in $\Delta \Psi_{\mathrm{m}}$ were closely linked to the activation of sarcolemmal $K_{A T P}$ current, which is a consequence of accelerated ATP hydrolysis by the uncoupled mitochondria, resulting in shortening or elimination of the cellular AP (8). The goal of the present study, therefore, was to determine if this mechanism contributes to alterations in the electrophysiology of hearts subjected to IR.

Benzodiazepines are widely used clinically for their central nervous system effects, which are primarily mediated through their interaction with central BzRs (e.g., the GABA/BzR complex; ref. 21). A second receptor for benzodiazepines is found in peripheral tissues (sometimes referred to as the peripheral BzR) and is abundant in the mitochondrial membranes (22) of most cells (thus referred to as $\mathrm{mBzR}$ herein). The pharmacology of the $\mathrm{mBzR}$ is distinct from the central receptor subtype, and it is selectively inhibited by the benzodiazepine Ro 5-4864, generically known as 4'-Cl-DZP, as well as the nonbenzodiazepine, isoquinoline carboxamide derivative 

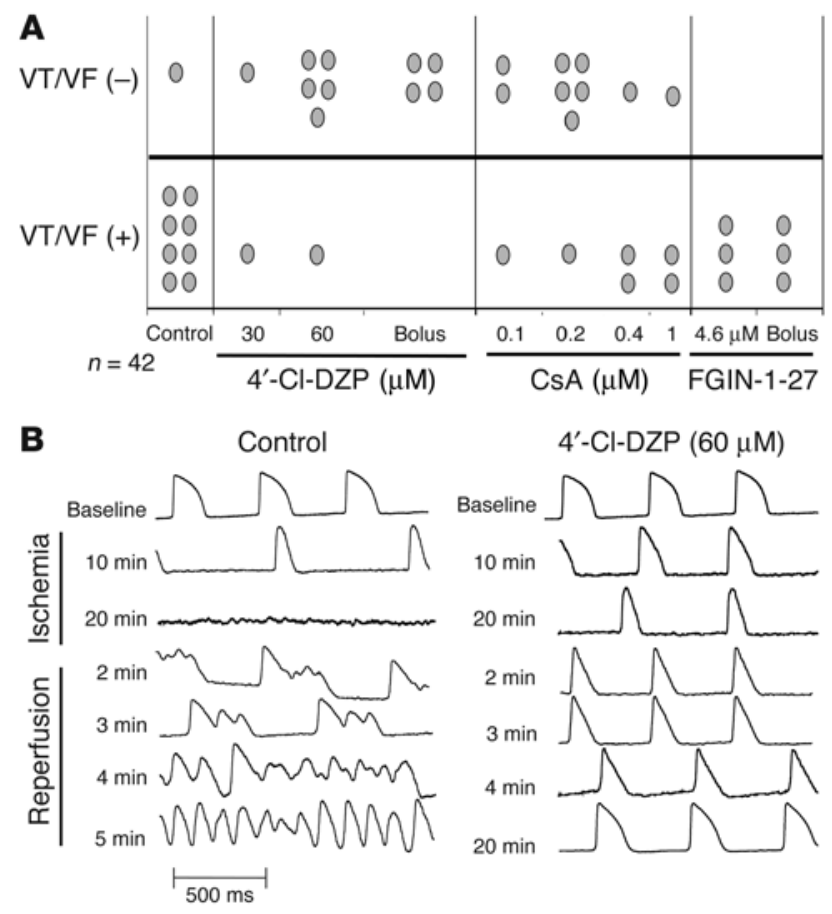

\section{Figure 6}

Effects of drugs on post-ischemic arrhythmias. (A) Incidence of reperfusion-related arrhythmias in all groups. (B) Representative AP traces recorded in a control heart (left) and a heart pretreated with 4 '-CI-DZP (right) at various time points during $\mathrm{IR}$, demonstrating the protection of 4'-Cl-DZP against reperfusion arrhythmias.

PK11195. Similarly, the agonist FGIN-1-27 (2-aryl-3-indoleacetamide) binds with high affinity to the $\mathrm{mBzR}$ but not to the central BzR (23). The mBzR inhibitors are known to block mitochondrial IMAC, as originally described in swelling assays of isolated mitochondria $(24,25)$, and were later shown to block anion channels in single-channel patch clamp studies of isolated mitoplasts (26). We have also confirmed that 5-50 $\mu \mathrm{M}$ concentrations of PK11195 and 4'-Cl-DZP block mitochondrial inner membrane ion channels in cardiac mitoplasts (data not shown). Importantly, we have previously demonstrated that either PK11195 or 4'-Cl-DZP could acutely block (or prevent if present beforehand) $\Delta \Psi_{\mathrm{m}}$ oscillations triggered by metabolic stress $(8,18)$ and that FGIN-1-27 promoted mitochondrial depolarization (8). These studies justified the use of these compounds to test our central hypothesis.

Consistent with the mitochondria being the main target of the $\mathrm{mBzR}$ antagonists, we demonstrate here that 4'-Cl-DZP had no effect on the AP in the intact perfused heart in the absence of metabolic stress, indicating that a direct effect of 4'-Cl-DZP on sarcolemmal ion channels was unlikely. When the mitochondria were stressed, however, and $\Delta \Psi_{\mathrm{m}}$ became unstable, $4^{\prime}$-Cl-DZP rapidly stabilized both $\Delta \Psi_{\mathrm{m}}$ and the cellular AP (Figure 1). Variations in $\Delta \Psi_{\mathrm{m}}$ and the $\mathrm{APD}_{90}$ were always highly correlated due to activation of sarcolemmal $\mathrm{K}_{\mathrm{ATP}}$ currents during mitochondrial uncoupling. We have previously shown that whole-cell $\Delta \Psi_{\mathrm{m}}$ oscillations in isolated cardiomyocytes are not suppressed by CsA (8) and do not depend on intracellular $\mathrm{Ca}^{2+}(18)$.

Effects of $m B z R$ inbibition on whole heart electrophysiology during $I R$. At the onset of ischemia, AP shortening occurs rapidly, presum- ably as a result of the activation of sarcolemmal $\mathrm{K}_{\mathrm{ATP}}$ channels due to the failure of oxidative phosphorylation and the inhibition of anaerobic metabolism. Relatively little is known about the time course of loss of $\Delta \Psi_{\mathrm{m}}$ during ischemia, but available evidence suggests that significant changes can occur within the first 5-10 minutes of interruption of perfusion. AP shortening and eventual electrical inexcitability occurred monotonically within 20 minutes of the onset of global ischemia in the guinea pig hearts, and this effect was blunted by equilibration of the heart with 4'-Cl-DZP in a concentration-dependent manner (Figure 2). Moreover, FGIN1-27 treatment markedly accelerated AP shortening and decreased the time to inexcitability (Figure 3A). These findings correspond to the effects of these compounds on $\Delta \Psi_{\mathrm{m}}$ and indicate that IMAC may be activated very early during ischemia. Surprisingly, although sarcolemmal $\mathrm{K}_{\mathrm{ATP}}$ channel activation has been postulated to play a protective role during ischemia by reducing cellular energy demand, in the present study we found that prolonging the duration of electrical excitability by 4'-Cl-DZP well into the ischemic period was correlated with improved AP recovery upon reperfusion. Indeed, the most striking effect of $4^{\prime}-\mathrm{Cl}-\mathrm{DZP}$ was to restore $\mathrm{APD}_{90}$ to near baseline levels within approximately 6 minutes of reperfusion (Figure 7C). Treatment with 4'-Cl-DZP and the facilitation of normal AP morphology were correlated with the prevention of postischemic tachyarrhythmias (Figures $6 \mathrm{~B}$ and 7). Notably, a single bolus dose of 4'-Cl-DZP given just prior to reperfusion was also effective at stabilizing the AP and preventing arrhythmias (Figure 6A), identifying a novel therapeutic strategy for preventing postischemic electrical dysfunction.

The mitochondrial PTP is thought to open during reperfusion but not during ischemia (10). Inhibiting this channel with CsA had no effect on APD shortening during ischemia but did influence AP recovery upon reperfusion (Figure 7). CsA-treated hearts exhibited a period of electrical silence followed by a delayed and incomplete recovery of AP, although the incidence of sustained arrhythmias was reduced (Figures $6 \mathrm{~A}$ and 7 ). The more rapid recovery of the $\mathrm{AP}$ in the presence of $4^{\prime}$-Cl-DZP suggests that there may be a hierarchy of mitochondrial channels activated by IR. IMAC activation during ischemia and early reperfusion may be a precursor to the opening of the mitochondrial PTP, which would mark an irreversible step toward cell death. Activation of IMAC (by FGIN-1-27) not only accelerated APD shortening during ischemia (Figure 3A) but prolonged electrical inexcitability upon reperfusion, ultimately leading to sustained ventricular tachyarrhythmias (Figure $7 \mathrm{~A})$. Thus, stabilization of $\Delta \Psi_{\mathrm{m}}$ early in the reperfusion phase by inhibiting the mBzR was found to be the most effective means of preventing VT and VF.

Metabolic sink/block as a novel mechanism of arrbythmogenesis. A number of existing paradigms have been put forward to explain electrical dysfunction in the heart. Triggered arrhythmias involving abnormal ventricular automaticity have generally been associated with the cellular mechanisms of early or delayed afterdepolarizations of the myocytes or ectopic foci that may develop near the border zone of an infarct. In contrast, reentrant arrhythmias are thought to depend on the macroscopic spatiotemporal conduction properties of the myocardium. Heterogeneous conduction block is a key feature of models of reentry (27). Block of electrical propagation in the myocardium has been attributed to 3 cellular mechanisms at the microscopic level: (a) loss of cellcell coupling by closure of gap junctions, (b) regional uncoupling by anatomical barriers to conduction (e.g., scar tissue), or 
A
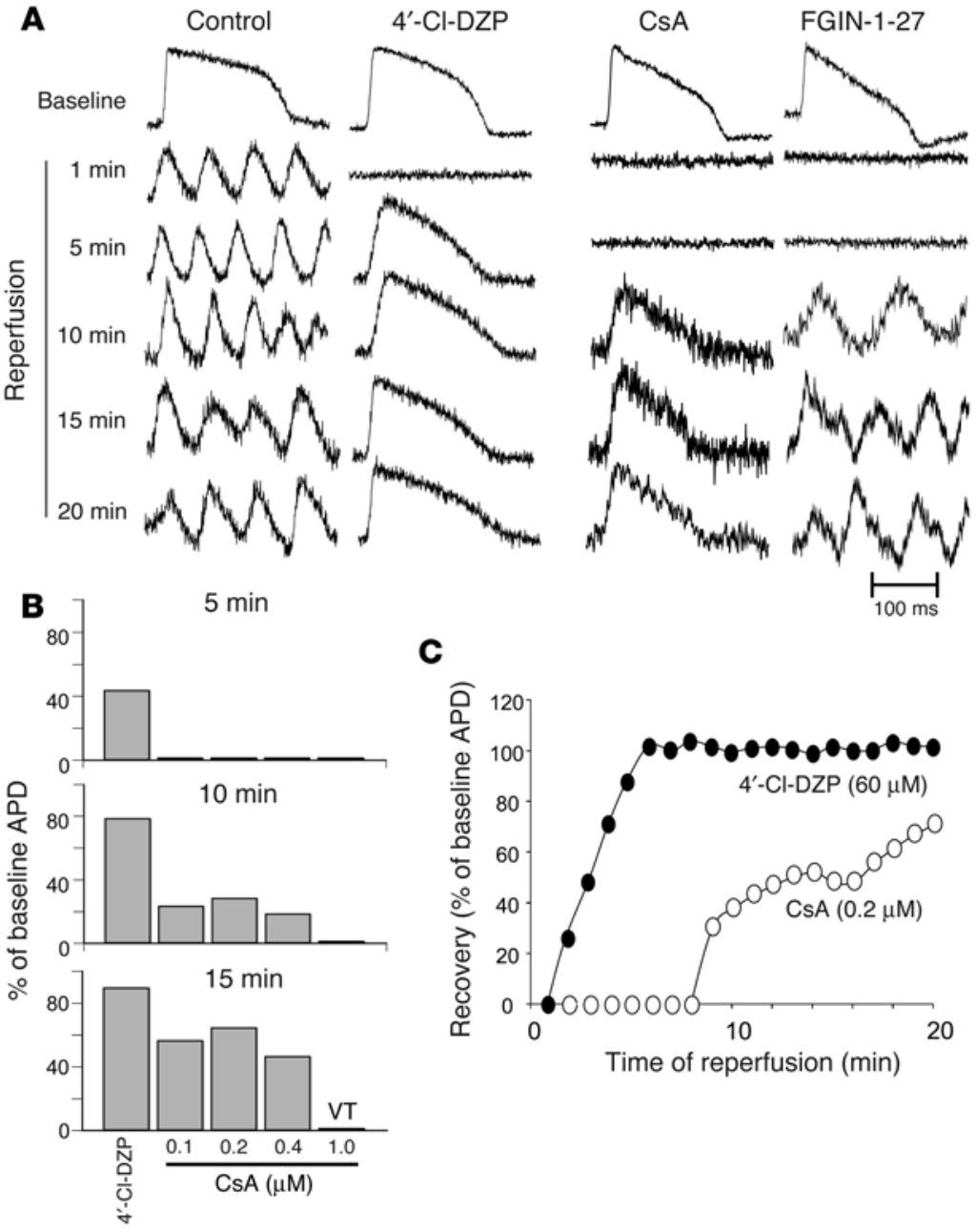

C

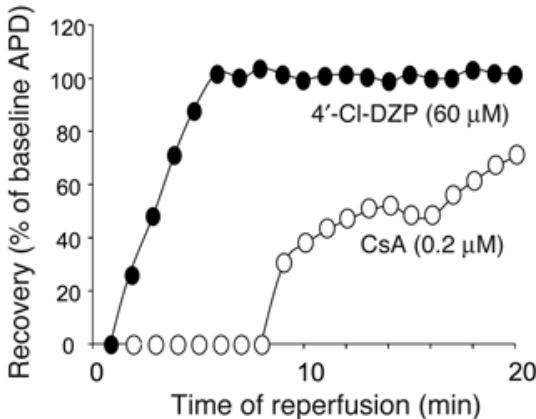

(c) dynamic functional block due to heterogeneity of intrinsic electrophysiological restitution properties.

Based on the present findings, we propose a fourth mechanism of conduction block and reentry dependent on the formation of areas of the myocardium undergoing regional or temporal changes in $\Delta \Psi_{\mathrm{m}}$, constituting a metabolic current sink. In this case, the propagating wave of depolarization encounters clusters of cells in which the mitochondrial network is depolarized and the sarcolemmal $\mathrm{K}_{\mathrm{ATP}}$ channels are open. These cells are rendered inexcitable because of the large background $\mathrm{K}^{+}$conductance locking the sarcolemmal membrane potential close to $\mathrm{E}_{\mathrm{k}}$ rather than by their inability to conduct current; i.e., they are powerful current sinks. Consistent with this notion is the disproportionate reduction in APA and $d F / d t$ recorded during ischemia in FGIN-1-27-treated hearts (Figure 4). This mechanism would be distinct from existing conduction block models in that the charge of the depolarizing cell would be dissipated into the metabolic sink, causing the impulse to decrement and block. In contrast, for conduction block dependent on compromised gap junction function, the charge of the depolarized cell builds up when the block is encountered due to the reduced electrotonic sink, and the higher voltage increases the likelihood that the wave of depolarization will bypass the region of block via an alternative conduction path. Thus, gap-junctional block can increase the "safety factor" for conduction (27) whereas metabolic

\section{Figure 7}

Post-ischemic AP recovery and arrhythmias. (A) Representative APs during recovery upon reperfusion in control, 4'-Cl-DZP-, CsA-, and FGIN-1-27-treated hearts. (B) Recovery of APD after 5 minutes of reperfusion as a percentage of baseline APD in hearts treated with $64 \mu \mathrm{M} 4$ '-Cl-DZP and various concentrations of CsA, indicating that the optimal concentration was $0.2 \mu \mathrm{M}$. (C) Plot of the recovery of APD upon reperfusion normalized to the baseline APD before ischemia in $4^{\prime}-\mathrm{Cl}-$ DZP- and CsA-treated hearts.

sink/block dramatically decreases the safety factor. The latter could lead to a zone of functional block (see, for example, Figure 5 and Supplemental Video 3) that extends even beyond the regions containing myocytes with depolarized mitochondria.

In support of the metabolic sink/block hypothesis, the mBzR agonist FGIN-1-27, which promotes $\Delta \Psi_{\mathrm{m}}$ depolarization (8), not only accelerated APD shortening but created widespread regions of slowed conduction. Further investigation will be required to determine if decreased gap-junctional conductance might also be induced by the collapse of $\Delta \Psi_{\mathrm{m}}$ in order to distinguish between the 2 possible mechanistic explanations for impaired electrical propagation. In this regard, it should be noted that APD shortening as well as reduced APA and $d F / d t$ occurring during the first 10 minutes of ischemia were blunted by $\mathrm{K}_{\mathrm{ATP}}$ channel blockade as were the effects of FGIN-1-27 on the ischemic response (Figure 4), indicating that the opening of $\mathrm{K}_{\text {ATP }}$ channels, secondary to $\Delta \Psi_{\mathrm{m}}$ depolarization, was responsible for the early electrophysiological remodeling during ischemia. Electrical excitability beyond 20 minutes of ischemia, however, could only be preserved by blocking the upstream mitochondrial target, suggesting that prevention of energy depletion might also prevent changes occurring later in the ischemic period, which could include changes in gap junctional conductance. Inhibition of $\Delta \Psi_{\mathrm{m}}$ depolarization with $4^{\prime}$-Cl-DZP was also found to be more effective than continuous inhibition of $\mathrm{K}_{\mathrm{ATP}}$ channels in preventing reperfusion-induced tachyarrhythmias although a bolus dose of GLIBEN given just prior to reperfusion successfully prevented tachyarrhythmias in 2 of 3 hearts (data not shown). These findings emphasize the primacy of blocking the mechanism of mitochondrial de-energization above that of inhibiting the downstream effects of metabolism on ion channels. This argument can be extended further to the induction of the mitochondrial PTP; inhibition of this late-stage effector was able to restore electrical function only partially when compared with the effects of 4'-Cl-DZP (Figure 7C).

Regardless of the precise details of the initiation of reentry, the results strongly suggest that macroscopic electrical heterogeneity in the postischemic heart stems in part from instability at the subcellular level. That is, a perturbation of mitochondrial function can lead to the following 4 things: (a) failure of the mitochondrial network of the myocyte, (b) regional or temporal alterations in AP, (c) development of zones of impaired conduction, and (d) ultimately, a fatal ventricular arrhythmia. 
Conclusion. The present findings support the hypothesis that mitochondrial criticality plays a key role in the recovery of electrical activity in the postischemic heart. Blocking mitochondrial inner membrane ion channels through $\mathrm{mBzR}$ inhibition could prevent mitochondrial ROS-induced ROS release and the loss of $\Delta \Psi_{\mathrm{m}}$ triggered by metabolic stress. This effect of the $\mathrm{mBzR}$ antagonist was correlated with preservation of the AP during ischemia as well as restoration of normal electrical activity upon reperfusion. The nearly complete suppression of reperfusion arrhythmias by stabilization of $\Delta \Psi_{\mathrm{m}}$ represents a novel mechanistic advance toward understanding the basis of ischemia-related arrhythmias, and also suggests a powerful new therapeutic strategy for preventing sudden cardiac death in the context of acute coronary events.

\section{Methods}

Experimental preparation. All procedures involving the handling of animals were approved by the Animal Care and Use Committee of the Johns Hopkins University and adhered with NIH public health service guidelines. As described in detail elsewhere (28), adult guinea pigs $(n=48)$ were anesthetized with pentobarbital sodium ( $30 \mathrm{mg} / \mathrm{kg}$ i.p.) and, in accordance with the Lagendorff preparation, retrogradely perfused via the aorta with oxygenated $\left(95 \% \mathrm{O}_{2}-5 \% \mathrm{CO}_{2}\right)$ Tyrode solution containing $130 \mathrm{mM} \mathrm{NaCl}, 1.2 \mathrm{mM}$ $\mathrm{MgSO}_{4}, 4.75 \mathrm{mM} \mathrm{KCl}, 5.0 \mathrm{mM}$ dextrose, and $1.25 \mathrm{mM} \mathrm{CaCl}_{2}$ (pH 7.40) at $36 \pm 1{ }^{\circ} \mathrm{C}$. Hearts were stained with the voltage-sensitive dye di-4-ANEPPS $(15 \mu \mathrm{M}$; Molecular Probes Inc.) for 10 minutes and then positioned in a chamber such that the mapping field was centered over a $0.8-\mathrm{cm}$ diameter region of left ventricular epicardium midway between apex and base. Gentle pressure was applied to the posterior surface of the heart with a movable piston to stabilize the mapped surface against the imaging window of the chamber, allowing us to avoid the use of electromechanical inhibitors, which interfere with repolarization, and the incidence of arrhythmias. Although experiments were typically completed within 1.5 to 2 hours, these preparations remained stable for over 4 hours of perfusion. Pacing with a silver pin needle placed on the epicardium was performed intermittently and briefly at various times during the experimental protocol in order to obtain a quantitative assessment of epicardial CV at each time point. Pacing was then turned off in order to determine the intrinsic response of hearts to the IR protocol independent of exogenous stimulation.

High-resolution optical mapping. An optical mapping system was designed with the capability of simultaneously recording transmembrane APs from 464 sites. Emitted fluorescence was collected using a custom-designed optical macroscope imaging system consisting of a high numerical aperture photographic lens, a dichroic mirror, and an emission filter (Chroma Inc.) as previously described in detail (29). Emitted light exiting the detector lens was filtered (>610 $\mathrm{nm}$ ) and focused onto the photodiode array (WuTech Inc.). Photocurrent from each photodiode underwent current-tovoltage conversion, amplification, band-pass filtering, multiplexing, and digitization (1600 samples/s/channel) with 16-bit precision.

IR protocol. Following extraction and staining with di-4-ANEPPS, hearts were stabilized for 30 minutes. Monitoring of APs, perfusion pressure, coronary flow, and temperature ensured electrical stability in all preparations $(n=48)$ used in this study. Pharmacological agents were delivered to the heart in 1 of 2 ways: (a) continuously during control perfusion and reperfusion, or (b) in a high-dose bolus (5-10 $\times$ the optimal equilibration concentration) injected into the coronaries during the ischemic phase 5 minutes before the onset of reperfusion. After stabilization, hearts were either perfused with control tyrode solution (control group) or with tyrode containing the $\mathrm{mBzR}$ antagonist 4'-Cl-DZP (32-100 $\mu \mathrm{M})$, the mBzR agonist FGIN-1-27 (4.6-46 $\mu \mathrm{M})$, the PTP inhibitor CsA $(0.1-1.0 \mu \mathrm{M})$, or the sarcolemmal $\mathrm{K}_{\text {ATP }}$ channel blocker GLIBEN $(10 \mu \mathrm{M})$ for 25 minutes. Following baseline perfusion at
$15-20 \mathrm{ml} / \mathrm{min}$ to maintain a perfusion pressure of $60-70 \mathrm{mmHg}$, flow was completely stopped for 30 minutes (ischemia phase), and APs (3.8-second epochs) were recorded at 1 -minute intervals. Following the ischemia phase, perfusion was restored initially at the same flow rate and then was adjusted slightly to exactly match the perfusion pressure during the baseline perfusion phase (reperfusion phase). The optimal equilibration concentration of each agent was determined by its effects on ischemia-induced AP shortening, recovery of $\mathrm{APD}$, and/or prevention of arrhythmias upon reperfusion.

In a subset of experiments, 4'-Cl-DZP $(320 \mu \mathrm{M}, n=4)$, FGIN-1-27 (460 $\mu \mathrm{M}, n=3)$, or CsA $(1.0 \mu \mathrm{M}, n=3)$ were delivered to the heart in the form of a high-concentration bolus injection delivered directly into the cannula via a side port.

Cardiomyocyte isolation. Cellular electrophysiological measurements were performed on freshly isolated adult guinea pig ventricular myocytes prepared by enzymatic dispersion, as previously described (17). Imaging and electrophysiological recordings were performed after suspending the cells in a solution containing $140 \mathrm{mM} \mathrm{NaCl}, 5 \mathrm{mM} \mathrm{KCl}, 1 \mathrm{mM} \mathrm{MgCl}_{2}, 10 \mathrm{mM}$ HEPES, and $1 \mathrm{mM} \mathrm{CaCl}_{2}$, pH 7.4 (adjusted with $\mathrm{NaOH}$ ), supplemented with $10 \mathrm{mM}$ glucose. The dish containing the cardiomyocytes was equilibrated at $37^{\circ} \mathrm{C}$ with unrestricted access to atmospheric oxygen on the stage of a Nikon E600FN upright microscope.

Two-photon laser scanning microscopy. The cationic potentiometric fluorescent dye TMRM was used to monitor changes in $\Delta \Psi_{\mathrm{m}}$ as previously described (8). Images were recorded using a 2-photon laser scanning microscope (Bio-Rad MRC-1024MP; Bio-Rad Laboratories) with excitation at $740 \mathrm{~nm}$ (Tsunami Ti:Sa laser; Spectra-Physics), and the red emission of TMRM was collected at $605 \pm 25 \mathrm{~nm}$. Other imaging conditions were as previously described (8). Images were analyzed offline using ImageJ software (Wayne Rasband, NIH; http://rsb.info.nih.gov/ij/).

Cardiomyocyte electrophysiological studies. Freshly isolated ventricular myocytes, handled as described above, were whole-cell patch clamped in a flow chamber on the stage of the 2-photon microscope using borosilicate glass pipettes (1-4 M $\Omega$ tip resistance). APs were recorded in current clamp mode using an Axopatch 200A amplifier coupled to a Digidata 1200A interface (Axon Instruments), as previously described (8). Myocytes were superfused with $140 \mathrm{mM} \mathrm{NaCl}, 5 \mathrm{mM} \mathrm{KCl}, 1 \mathrm{mM} \mathrm{MgCl}, 2 \mathrm{mM} \mathrm{CaCl}_{2}, 10 \mathrm{mM}$ glucose, and $10 \mathrm{mM}$ HEPES (pH 7.4 with $\mathrm{NaOH}$ ). Intracellular solutions contained $130 \mathrm{mM}$ potassium glutamate, $9 \mathrm{mM} \mathrm{KCl}, 10 \mathrm{mM} \mathrm{NaCl}, 0.5 \mathrm{mM} \mathrm{MgCl}_{2}$, 5 mM MgATP, 1 mM EGTA, and 10 mM HEPES (pH 7.0 with KOH). APs were evoked by brief $(5 \mathrm{~ms})$ current injections applied at 2-second intervals.

Materials. TMRM and di-4-ANEPPS were purchased from Invitrogen Corp. 4'-Cl-DZP, FGIN-1-27, CsA, and GLIBEN were obtained from Sigma-Aldrich. Stock solutions of these reagents were prepared in DMSO and concentrated enough to avoid exceeding $0.1 \% \mathrm{DMSO}(\mathrm{v} / \mathrm{v})$ in the final solution.

Statistics. Comparisons were performed using the unpaired, 1-tailed Student's $t$ test. Data were considered significantly different at $P<0.05$.

\section{Acknowledgments}

This work was supported by NIH grants R01-HL54598 (to B. O’Rourke) and P50-HL52307 (to G.F. Tomaselli).

Received for publication April 15, 2005, and accepted in revised form August 30, 2005.

Address correspondence to: Brian O'Rourke, Division of Cardiology, Johns Hopkins University, 720 Rutland Avenue, Ross 844, Baltimore, Maryland 21205, USA. Phone: (410) 614-0034; Fax: (410) 955-7953; E-mail: bor@jhmi.edu.

Fadi G. Akar and Miguel A. Aon contributed equally to this work. 
1. Carmeliet, E. 1999. Cardiac ionic currents and acute ischemia: from channels to arrhythmias. Physiol. Rev. 79:917-1017.

2. Coronel, R., et al. 1992. Reperfusion arrhythmias in isolated perfused pig hearts. Inhomogeneities in extracellular potassium, ST and TQ potentials, and transmembrane action potentials. Circ. Res. 71:1131-1142.

3. De Groot, J.R., and Coronel, R. 2004. Acute ischemia-induced gap junctional uncoupling and arrhythmogenesis. Cardiovasc. Res. 62:323-334.

4. Picard, S., et al. 1999. KATP channels and 'border zone' arrhythmias: role of the repolarization dispersion between normal and ischaemic ventricular regions. Br. J. Pharmacol. 127:1687-1695.

5. Crompton, M., Virji, S., Doyle, V., Johnson, N., and Ward, J.M. 1999. The mitochondrial permeability transition pore. Biochem. Soc. Symp. 66:167-179.

6. Duchen, M.R. 1999. Contributions of mitochondria to animal physiology: from homeostatic sensor to calcium signalling and cell death. J. Physiol. 516:1-17.

7. Romashko, D.N., Marban, E., and O'Rourke, B. 1998. Subcellular metabolic transients and mitochondrial redox waves in heart cells. Proc. Natl. Acad. Sci. U. S. A. 95:1618-1623.

8. Aon, M.A., Cortassa, S., Marban, E., and O'Rourke, B. 2003. Synchronized whole cell oscillations in mitochondrial metabolism triggered by a local release of reactive oxygen species in cardiac myocytes. J. Biol. Chem. 278:44735-44744.

9. Ambrosio, G., et al. 1993. Evidence that mitochondrial respiration is a source of potentially toxic oxygen free radicals in intact rabbit hearts subjected to ischemia and reflow. J. Biol. Chem. 268:18532-18541.

10. Griffiths, E.J., Ocampo, C.J., Savage, J.S., Stern, M.D., and Silverman, H.S. 2000. Protective effects of low and high doses of cyclosporin A against reoxygenation injury in isolated rat cardiomyocytes are associated with differential effects on mitochondrial calcium levels. Cell Calcium. 27:87-95.

11. Suleiman, M.S., Halestrap, A.P., and Griffiths, E.J. 2001. Mitochondria: a target for myocardial protection. Pharmacol. Ther. 89:29-46.

12. Kroemer, G., Dallaporta, B., and Resche-Rigon, M. 1998. The mitochondrial death/life regulator in apoptosis and necrosis. Annu. Rev. Physiol. 60:619-642.

13. Griffiths, E.J., and Halestrap, A.P. 1993. Protection by cyclosporin A of ischemia/reperfusion-induced damage in isolated rat hearts. J. Mol. Cell. Cardiol. 25:1461-1469.

14. Halestrap, A.P., Connern, C.P., Griffiths, E.J., and Kerr, P.M. 1997. Cyclosporin A binding to mitochondrial cyclophilin inhibits the permeability transition pore and protects hearts from ischaemia/reperfusion injury. Mol. Cell. Biochem. 174:167-172.

15. Weiss, J.N., Korge, P., Honda, H.M., and Ping, P. 2003. Role of the mitochondrial permeability transition in myocardial disease. Circ. Res. 93:292-301.

16. Berkich, D.A., Salama, G., and LaNoue, K.F. 2003. Mitochondrial membrane potentials in ischemic hearts. Arch. Biochem. Biophys. 420:279-286.

17. O'Rourke, B., Ramza, B.M., and Marban, E. 1994 Oscillations of membrane current and excitability driven by metabolic oscillations in heart cells. Science. 265:962-966.

18. O'Rourke, B. 2000. Pathophysiological and protective roles of mitochondrial ion channels. J. Physiol. 529:23-36.

19. Cortassa, S., Aon, M.A., Winslow, R.L., and O'Rourke, B. 2004. A mitochondrial oscillator dependent on reactive oxygen species. Biophys. J. 87:2060-2073.
20. Aon, M.A., Cortassa, S., and O’Rourke, B. 2004. Percolation and criticality in a mitochondrial network. Proc. Natl. Acad. Sci. U. S. A. 101:4447-4452.

21. DeLorey, T.M., and Olsen, R.W. 1992. Gamma-aminobutyric acidA receptor structure and function. J. Biol. Chem. 267:16747-16750.

22. Garnier, M., et al. 1993. Diazepam binding inhibitor is a paracrine/autocrine regulator of Leydig cell proliferation and steroidogenesis: action via peripheral-type benzodiazepine receptor and independent mechanisms. Endocrinology. 132:444-458.

23. Romeo, E., et al. 1992. 2-Aryl-3-indoleacetamides (FGIN-1): a new class of potent and specific ligands for the mitochondrial DBI receptor (MDR). J. Pharmacol. Exp. Ther. 262:971-978.

24. Beavis, A.D. 1992. Properties of the inner membrane anion channel in intact mitochondria. J. Bioenerg. Biomembr. 24:77-90.

25. Beavis, A.D., and Davatol-Hag, H. 1996. The mitochondrial inner membrane anion channel is inhibited by DIDS. J. Bioenerg. Biomembr. 28:207-214.

26. Kinnally, K.W., et al. 1993. Mitochondrial benzodiazepine receptor linked to inner membrane ion channels by nanomolar actions of ligands. Proc. Natl. Acad. Sci. U. S. A. 90:1374-1378.

27. Kleber, A.G., and Rudy, Y. 2004. Basic mechanisms of cardiac impulse propagation and associated arrhythmias. Physiol. Rev. 84:431-488.

28. Akar, F.G., Roth, B.J., and Rosenbaum, D.S. 2001. Optical measurement of cell-to-cell coupling in intact heart using subthreshold electrical stimulation. Am. J. Physiol. Heart Circ. Physiol. 281:H533-H542.

29. Akar, F.G., Spragg, D.D., Tunin, R.S., Kass, D.A., and Tomaselli, G.F. 2004. Mechanisms underlying conduction slowing and arrhythmogenesis in nonischemic dilated cardiomyopathy. Circ. Res. 95:717-725. 